\title{
BMJ Open Industry ties and evidence in public comments on the FDA framework for modifications to artificial intelligence/ machine learning-based medical devices: a cross sectional study
}

\author{
James Andrew Smith (D) ,1,2 Roxanna E Abhari (D) , 'Zain Hussain (iD) , ${ }^{3}$ \\ Carl Heneghan (D) , ${ }^{4}$ Gary S Collins (D) , ${ }^{1,5}$ Andrew J Carr (D) ${ }^{1,2}$
}

To cite: Smith JA, Abhari RE, Hussain Z, et al. Industry ties and evidence in public comments on the FDA framework for modifications to artificial intelligence/ machine learning-based medical devices: a cross sectional study. BMJ Open 2020;10:e039969. doi:10.1136/ bmjopen-2020-039969

- Prepublication history for this paper is available online To view these files, please visit the journal online (http://dx.doi. org/10.1136/bmjopen-2020039969).

Received 30 April 2020 Revised 20 July 2020 Accepted 21 July 2020

Check for updates

(c) Author(s) (or their employer(s)) 2020. Re-use permitted under CC BY. Published by BMJ.

For numbered affiliations see end of article.

Correspondence to Dr James Andrew Smith; james.smith2@ndorms.ox.ac.uk

\section{ABSTRACT}

Objectives To determine the extent and disclosure of financial ties to industry and use of scientific evidence in comments on a US Food and Drug Administration (FDA) regulatory framework for modifications to artificial intelligence/machine learning (Al/ML)-based software as a medical device (SaMD).

Design Cross-sectional study.

Setting We searched all publicly available comments on the FDA 'Proposed Regulatory Framework for Modifications to Artificial Intelligence/Machine Learning (Al/ML)-Based Software as a Medical Device (SaMD)_Discussion Paper and Request for Feedback' from 2 April 2019 to 8 August 2019.

Main outcome measures The proportion of articles submitted by parties with financial ties to industry, disclosing those ties, citing scientific articles, citing systematic reviews and meta-analyses, and using a systematic process to identify relevant literature. Results We analysed 125 comments submitted on the proposed framework. $79(63 \%)$ comments came from parties with financial ties; for $36(29 \%)$ comments, it was not clear and the absence of financial ties could only be confirmed for $10(8 \%)$ comments. No financial ties were disclosed in any of the comments that were not from industry submitters. The vast majority of submitted comments (86\%) did not cite any scientific literature, just $4 \%$ cited a systematic review or meta-analysis and no comments indicated that a systematic process was used to identify relevant literature.

Conclusions Financial ties to industry were common and undisclosed, and scientific evidence, including systematic reviews and meta-analyses, were rarely cited. To ensure regulatory frameworks best serve patient interests, the FDA should mandate disclosure of potential conflicts of interest (including financial ties) in comments, encourage the use of scientific evidence, and encourage engagement from non-conflicted parties.

\section{INTRODUCTION}

Artificial intelligence and machine learning (AI/ML) are increasingly prevalent in the healthcare literature. ${ }^{1}$ At least 14 medical
Strengths and limitations of this study

- We analysed the extent of financial ties to industry and the use of scientific evidence in comments on the proposed Food and Drug Administration (FDA) framework.

- We used a comprehensive strategy to attempt to identify financial ties to industry.

There is heterogeneity in the degree of conflict with respect to the framework that the recorded financial ties represent; some ties are more likely than others to result in biased commenting.

- Because the framework could not be classified as pro-industry or not, we did not classify the direction of opinions expressed in comments with respect to the framework and their association with financial ties.

- We do not know how information submitted to FDA is used internally in the rule-making process, and, therefore, how the identified financial ties will impact the regulation.

devices incorporating $\mathrm{AI} / \mathrm{ML}$ have now been cleared by the US Food and Drug Administration (FDA), ${ }^{2}$ including an autonomous diagnostic system for diabetic retinopathy that does not require input from a clinician for interpretation. ${ }^{3}$ Because AI may learn and adapt to additional data in real time to improve performance, regulators have questioned the suitability of traditional medical device regulatory pathways for AI/ ML containing devices. Changes that might affect a device's performance under the current regulatory framework would require further review from the FDA, which is timeconsuming and may not suit the iterative modification that often characterises software development and deployment. The FDA has therefore proposed a regulatory framework for modifications to AI/ML-based software 
Box 1 Summary of AI/ML framework: 'Proposed

Regulatory Framework for Modifications to Artificial

Intelligence/Machine Learning (AI/ML)-Based Software as

a Medical Device (SaMD)—Discussion Paper and Request for Feedback ${ }^{34}$

The FDA released the first discussion paper on 2 April 2019 outlining a framework for regulating modifications to SaMD that use AI ML. The comment period closed on 3 June 2019. The proposed framework describes:

- The extent to which FDA's traditional framework for assessing modifications to devices should apply to Al/ML SaMD.

- Modification categories for continuously learning AI/ML SaMD and a proposed 'pre-determined change control plan' in pre-market submissions: when seeking regulatory approval, manufacturers would also submit a plan for modifications, including model retraining, as part of the initial pre-market review.

- Expectations for manufacturers to monitor the real-world performance of $\mathrm{Al} / \mathrm{ML}$ systems and periodically report updates to users and to the FDA on what changes have been implemented.

- The evaluation, monitoring and management of risks from $\mathrm{Al} / \mathrm{ML}$ modifications from initial pre-market submission through to postmarket performance.

- Hypothetical examples of modifications and their applicability to the proposed framework.

as a medical device (SaMD) ${ }^{4}$ (box 1). Because the FDA is one of the most prominent regulatory agencies, other agencies may follow FDA regulatory approaches. It is therefore essential that the framework reflects and promotes patient interests and safety.

Agencies base decisions on sound reasoning and scientific evidence, ${ }^{5}$ and the process of developing FDA regulations and guidance involves opportunities for the public to assess and comment on proposed rules before they are finalised. Comments can be submitted by anyone and are considered by the FDA in subsequent drafts and final rulings. However, there is potential for financial conflicts of interest (COI) among commenters, who could serve to benefit from particular outcomes such as less stringent regulatory requirements. Therefore, we evaluated the prevalence and disclosure of financial ties to industry in comments on the recent proposed $\mathrm{AI} / \mathrm{ML}$ device framework. There is a huge academic literature available related to AI/ML, which could be used to inform the development of new regulations, and the FDA explicitly looks for 'good science' in submitted comments. ${ }^{6}$ We also, therefore, examined the citation of scientific evidence, including of systematic reviews, to determine whether there is opportunity to increase and improve its use in comments.

\section{METHODS}

The docket folder for comment submission for the 'Proposed Regulatory Framework for Modifications to Artificial Intelligence/Machine Learning (AI/ML)-Based Software as a Medical Device (SaMD)_Discussion Paper

\begin{tabular}{|c|c|}
\hline Category & Explanation \\
\hline Academia & $\begin{array}{l}\text { Included individual academics and academic } \\
\text { groups }\end{array}$ \\
\hline Healthcare & $\begin{array}{l}\text { Included healthcare associations and health } \\
\text { professionals }\end{array}$ \\
\hline Industry & $\begin{array}{l}\text { Included companies, industry associations and } \\
\text { individuals from industry }\end{array}$ \\
\hline $\begin{array}{l}\text { Individual } \\
\text { consumers }\end{array}$ & $\begin{array}{l}\text { Category only used when provided in the } \\
\text { submitter information }\end{array}$ \\
\hline $\begin{array}{l}\text { Mixed } \\
\text { associations }\end{array}$ & $\begin{array}{l}\text { Defined as associations comprising at least } \\
\text { one industry and non-industry organisation }\end{array}$ \\
\hline $\begin{array}{l}\text { Federal } \\
\text { government }\end{array}$ & $\begin{array}{l}\text { Category only used when provided in the } \\
\text { submitter information }\end{array}$ \\
\hline Spam & $\begin{array}{l}\text { Recorded when the comment content was } \\
\text { clearly not related to the content of the FDA } \\
\text { document }\end{array}$ \\
\hline Other & $\begin{array}{l}\text { Recorded when the submitter was not one } \\
\text { of the above categories but was identifiable. } \\
\text { An example was the US Technology Policy } \\
\text { Committee of the Association for Computing } \\
\text { Machinery }\end{array}$ \\
\hline Unknown & $\begin{array}{l}\text { Recorded when no category was listed in the } \\
\text { submitter information, insufficient information } \\
\text { was provided to identify the submitter category } \\
\text { (ie, a name, but no affiliation was provided) } \\
\text { and when the commenter was listed as } \\
\text { 'Anonymous'. }\end{array}$ \\
\hline
\end{tabular}

FDA, Food and Drug Administration.

and Request for Feedback, ${ }^{7}$ was accessed 8 August 2019 and meta-data for all comments from 2nd April 2019 (when the discussion paper was published) to 8th August 2019 were exported to Microsoft Excel. Any comments submitted after this date, which was after the comment period closed, were not included in analysis. Individual comments were subsequently accessed via the docket by following the link in the Excel export. We conducted a pilot study in which JAS developed a data extraction protocol by reading and preliminarily analysing all comments submitted on the proposed framework. Two reviewers (REA and $\mathrm{ZH}$ ) then independently extracted data from all comments according to the extraction protocol (available on Open Science Framework: https:/ / osf.io/g423d/). We accessed the data from August to October 2019 and the comment order was randomised for each reviewer. JAS consolidated any discrepancies between the reviewers. Some minor changes were made to the data extraction procedure during the consolidation process which are described in the extraction protocol. JAS extracted and checked any new data as required.

\section{Category}

Comment submitters were categorised according to the categories in table 1 . Some submitters provide a 'Category' in the 'Submitter Information' section of the docket folder. When the category could not be determined by 
the extractor, the category provided in the submitter information was used, if provided.

\section{Financial ties to industry}

We searched for financial ties among comment submitters. A financial tie was defined as a financial link with industry. Submitters in the industry or mixed association category were assumed to have an industry tie. For other submitters, we determined whether there were financial ties according to the following method:

1. If the comment submitter was an academic (or group of academics), we searched the name of the academic(s) plus their institution to find academic pages or pages mentioning them on industry websites and determined if they were advisors or board members for industry, or had another obvious link. If this was not the case, we examined the two most recent publications (published from July 2017 until October 2019) that we could identify for each author and checked for industry affiliations and disclosures of personal fees, speaking fees, board-membership, employment, grants or similar from industry or industry associations. If at least one author of the comment had a financial tie according to these criteria, we recorded that there was a financial tie. If, of the two most recent publications, at least one stated that there was no financial tie or COI to disclose (or similar wording), and the other did not disclose a financial tie, we recorded no financial tie. If only one paper was identifiable, this was deemed sufficient to identify a conflict or lack thereof (ie, if only one paper was found and this stated there was no conflict, we recorded 'no').

2. For individual consumers or health professionals that provided their institution or another means of identifying them, we followed step 1 and additionally searched the open payments database (https://openpaymentsdata.cms.gov/) for their name and looked for contributions from industry of any sort from July 2017 to October 2019. A financial tie was recorded if the submitter had received contributions from industry and we were able to verify that the individual in the database was the commenter, for example, by cross-referencing their institution.

3. For healthcare associations and 'other' submitters, we searched for financial ties according to step 2 among all of the authors listed on the comment and, if no authors were listed, among board members. If any authors, or at least half of the board members had financial ties, the submitter was considered to have a financial tie. When no financial tie could be identified for an association, we recorded that there was none.

4. For submitters in the federal government category, we assumed that there was no financial tie.

5 . When the presence or absence of a financial tie could not be determined according to these criteria, it was recorded as unknown.

\section{Disclosure of financial ties}

For industry submitters, we assumed that disclosure would not be required because the potential COI is self-evident, but that for any other potentially conflicted submitters, a disclosure would be required for the FDA to be aware of the financial tie. Therefore, for non-industry submissions with financial ties, we recorded whether or not ties were disclosed.

\section{Scientific evidence and systematic searches}

As a proxy for the use of scientific evidence in comments, we recorded whether comments cited scientific articles, systematic reviews or meta-analyses, and whether any systematic search was reported to identify any documents or literature. We identified articles by reading each comment and examining footnotes, bibliographies or in-text citations, and considered academic journal articles or preprints/conference papers (eg, papers on ArXiv or IEEE) to be scientific articles. To identify citations of systematic reviews or meta-analyses, we looked at the titles of any referenced articles for the terms (or similar terms to) 'systematic review' or 'meta-analysis' and, where it was unclear, we reviewed the abstract or if necessary the full text. We determined whether or not a systematic search was used to identify documents or literature referenced or discussed in the comment. A systematic search in this context refers to a process that would be repeatable by an independent reader, such as listing the search terms used to identify literature.

\section{Document length}

We recorded the length of each comment to give an indication of its comprehensiveness. Specifically, we recorded the length, in pages, of any of the attachments on the docket page for the comment. If multiple attachments were provided, we recorded sum of the lengths (unless attachments were duplicates, eg, one in word and one as a pdf, in which case we recorded non-duplicates). For comments that did not provide an attachment, we copied and pasted the comment into a word document with standard font size 12 and recorded the length in pages.

\section{Data analysis}

Duplicate, near duplicate and spam comments were excluded from analysis. All other comments were included. Analysis was descriptive and was conducted in R V.3.6.1. ${ }^{8}$ The R code and dataset are provided on Open Science Framework (https://osf.io/g423d/). The analysis was reproduced in Python independently by ZH.

\section{Patient and public involvement}

There was no patient and public involvement in this study.

\section{RESULTS}

One hundred and thirty public comments were submitted in response to the proposed regulatory framework at the time we downloaded data (8 August 2019), of which five were duplicates, near-duplicates or spam, leaving 125 
Table 2 Presence of financial interests, citation of scientific evidence and document length

\begin{tabular}{|c|c|c|c|}
\hline Financial ties to industry & $\%$ of total (n) & $\%$ citing scientific evidence $(n)^{*}$ & Median document length, pages (range) \\
\hline Yes† & $63.2(79)$ & $16.5(13)$ & $4(1-30)$ \\
\hline No & $8(10)$ & $10(1)$ & $2.5(1-13)$ \\
\hline
\end{tabular}

*Including articles in journals or preprints.

†Includes industry, industry associations, mixed associations and any other submitters for which we were able to identify a financial tie to industry.

comments for analysis. Combining industry, mixed associations (which include representatives of industry) and other contributors with financial ties revealed that at least $79(63 \%)$ comments came from parties with financial ties (table 2). For 36 comments (29\%), it was not clear, and the absence of a financial ties could be confirmed in only 10 comments $(8 \%)$. The length of comments and proportion of comments citing scientific evidence was similar across submitters regardless of financial interest (table 2).

Table 3 summarises the categories of organisations or individuals submitting comments. Industry submitted 64 $(51 \%)$ comments. Of the 61 non-industry comments, we were able to determine whether or not there were financial ties to industry in 25 submissions $(41 \%)$. Of these 25 , financial ties for 15 were identified $(60 \%)$, and there were none for 10 (40\%). No financial ties were disclosed in any of the non-industry comments.

Of the 125 comments, 108 (86\%) did not cite scientific literature, $15(12 \%)$ cited at least one paper published in an academic journal and $2(1.6 \%)$ cited article preprints but not papers in academic journals. Five (4\%) comments cited a systematic review or meta-analysis, and none of the 125 comments reported any systematic method for identifying the literature referenced in the document.

\section{DISCUSSION}

\section{Statement of principal findings}

Industry and other submitters with financial ties to industry comprised nearly two-thirds of the comments on the proposed FDA framework. We found no evidence that submitters without a financial interest were more likely to cite scientific evidence or contribute more comprehensive comments. Identifying financial ties was not straightforward and required extensive searching. For many comments, the presence of financial ties was unknown; it is not possible to identify financial ties for anonymous commenters, or those that provide a name but no organisation, for example. The absence of financial ties could be confirmed in very few cases, and disclosure of ties was non-existent. Scientific literature was rarely cited across all submissions, just five comments cited systematic reviews and meta-analyses, and no comments reported a systematic process for identifying the literature. There were far fewer academic submissions than those from industry.

\section{Strengths and limitations}

In terms of strengths, this study is the first, that we know of, to examine financial ties among commenters on a proposed FDA regulatory framework, and adds to the growing literature documenting the prevalence of industry interests in public comment processes. ${ }^{9}$ We used a thorough method for identification of financial ties which has identified more potentially conflicted parties than would be apparent from the self-identification of the commenters. We also examined the citation of scientific evidence, adding to literature documenting lack of scientific evidence in public comments in other contexts. ${ }^{10}$ The raw data from this study are available publicly, so that readers can view specific financial ties, evidence cited or reanalyse the data if desired. Finally, the AI/ML regulatory framework is still under development, and these

\begin{tabular}{|c|c|c|c|}
\hline Category* & $\%$ of total $(n)$ & $\%$ citing scientific evidence $(\mathrm{n}) \dagger$ & Median document length, pages (range) \\
\hline Industry & $51.2(64)$ & $12.5(8)$ & $4(1-30)$ \\
\hline Academia & $17.6(22)$ & $9.1(2)$ & $1(1-13)$ \\
\hline Healthcare & $9.6(12)$ & $16.7(2)$ & $2.5(1-8)$ \\
\hline Unknown & $7.2(9)$ & $0(0)$ & $1(1-6)$ \\
\hline Other & $5.6(7)$ & $57.1(4)$ & $2(1-5)$ \\
\hline Mixed association & $4(5)$ & $20(1)$ & $5(4-8)$ \\
\hline Individual consumer & $3.2(4)$ & $0(0)$ & $1(1)$ \\
\hline Federal government & $1.6(2)$ & $0(0)$ & $1.5(1-2)$ \\
\hline
\end{tabular}

*See table 1 for explanations of categories.

†Percentage of that 'Category' citing scientific evidence, including articles in journals or preprints. 
findings may motivate better disclosure and use of scientific evidence as it continues to be developed.

In terms of limitations, the prevalence of financial ties in our study (63\%) is likely to be an underestimate. For $29 \%$ (36) of commenters, we were unable to confirm the presence or absence of financial ties because insufficient information was provided to identify their presence or absence according to our search strategy. Therefore, $63 \%$ should be interpreted as an estimate of the minimum prevalence of industry ties. In addition, there is heterogeneity in the degree of conflict with respect to the framework that the recorded financial ties may represent; some ties will be more likely than others to result in biased commenting.

Relatedly, we did not classify the direction of opinions expressed in comments with respect to the framework and their association with financial ties, in contrast to other work in similar areas. ${ }^{112}$ Because of the early stage and diversity of topics proposed in the regulatory framework, it is not clear that supporting the framework would be putting financial considerations ahead of patient considerations, or vice versa. We are, therefore, only able to make any inferences regarding the prevalence of financial ties, and not their impact on the opinions expressed in comments with regards to considerations of health versus financial interests. This is an important limitation because we cannot assume that financial ties will lead to biased commenting or will necessarily represent COI. In some cases, industry ties or COI do not introduce bias and may be aligned with patient interest ${ }^{13}{ }^{14}$; however, in others, there is ample evidence that they do lead to bias. ${ }^{11} 1215-17$

We have access only to the information externally submitted to FDA by the public, and undoubtedly further evidence is considered internally. We also do not know how the information gathered from public consultations is used internally and how it will impact the future regulatory framework. Agencies do not make decisions simply based on a majority of votes, ${ }^{59}$ so prevalence of financial ties and lack of use of scientific evidence does not necessarily mean that decision making internally is biased. However, the purpose of public consultations is to gather information from the public to inform regulation. It is important that such information is high quality, reasoned, objective and transparent, where possible.

\section{Implications and recommendations}

We recommend that the FDA request a statement of interests in comments, which would help to further determine the extent of COI in future public consultations. They could also reject anonymous comments unless there is a clear reason that anonymity would improve comment quality. Currently, the regulations.gov 'Tips for submitting effective comments' document does not mention disclosure of COI or financial ties, ${ }^{5}$ nor does the FDA's information page on submitting comments. ${ }^{6}$ In the absence of this requirement, submitters should proactively disclose any COI. COI may influence drug approvals, ${ }^{15}$ policy making ${ }^{11}{ }^{12}$ and reporting of results in research, ${ }^{16}{ }^{17}$ and may therefore also be influential in the present context. Although disclosure will not necessarily change the interpretation of information, it is an important first step in improving transparency.

We encourage greater participation by non-conflicted parties and academics in the development of this framework in the future. Participation should come both from experts in the technology, as well as from the broader medical community that will use and evaluate it. FDA could proactively engage with, for example, journals with expertise in the relevant areas to notify readers when relevant legislation is drafted. However, the responsibility should not lie solely with FDA, but participation in the commenting process can also be driven and rewarded by academic departments or groups with relevant expertise. Continued input from associations and the greater use of scientific evidence, in particular that from systematic reviews, by all commenters, could be valuable. At present, there are few relevant systematic reviews for this purpose (though exceptions include Nagendran et $a l^{8}$ ), and further work in this area would be useful.

In other areas of medical device regulation, there is literature available evaluating or critiquing regulatory frameworks. ${ }^{19-21}$ However, once regulations have been developed, it is a laborious process for them to be changed. AI/ML regulation is in an early stage where scientific knowledge could be used prospectively to help to define an appropriate framework and reduce the probability of issues occurring in the future. The regulations.gov guidance on commenting states: 'Although public support or opposition may help guide important public policies, agencies make determinations for a proposed action based on sound reasoning and scientific evidence rather than a majority of votes. A single, well-supported comment may carry more weight than a thousand form letters. ${ }^{5}$ If high quality comments are provided in future developments of the proposed rules, supported by sound scientific evidence, they may well be weighted more highly in decision making than other comments and directly impact the framework under development.

\section{CONCLUSION}

We found that the prevalence of financial ties to industry in commenters was high. For nearly $30 \%$ of comments, we were unable to determine whether or not there was a financial tie, and disclosure of ties was non-existent. The proportion of academic submitters was relatively low, and the use of scientific evidence to support comments was sparse. We recommend that the FDA requires disclosure of potential COI, and encourages greater academic participation and use of scientific evidence in public comments. The generalisability of this work has not been determined, and future work could extend the investigation to other policy areas or jurisdictions. 


\section{Author affiliations}

${ }^{1}$ NDORMS, University of Oxford Medical Sciences Division, 0xford, Oxfordshire, UK ${ }^{2}$ National Institute for Health Research Oxford Biomedical Research Centre, Oxford, Oxfordshire, UK

${ }^{3}$ College of Medicine and Veterinary Medicine, University of Edinburgh, Edinburgh, Scotland, UK

${ }^{4}$ Primary Health Care, University of Oxford, Oxford, Oxfordshire, UK

${ }^{5}$ Centre for Statistics in Medicine, University of Oxford, Oxford, Oxfordshire, UK

\section{Twitter James Andrew Smith @JamesASmith92}

Contributors JAS conceived the study, conducted a pilot study, analysed the data in $\mathrm{R}$ and wrote the first draft of the manuscript and is the guarantor, and planned the study with REA and AJC. REA extracted data from public comments and contributed to the first draft. ZH extracted data from public comments and repeated the data analysis independently in Python. In terms of reporting, all authors (JAS, REA, ZH, CH, GC, AJC) interpreted the data and reviewed and critically appraised the manuscript for important intellectual content. The corresponding author attests that all listed authors meet authorship criteria and that no others meeting the criteria have been omitted.

Funding This study was conducted as part of JAS' employment as a postdoctoral scientist funded by the National Institute for Health Research (NIHR) Oxford Biomedical Research Centre (BRC). Dr Heneghan holds grant funding from the NIHR Oxford BRC and the NIHR School of Primary Care Research Evidence Synthesis Working Group (Project 390). Dr Collins reports grants from Cancer Research Programme Grant (C49297/A27294), grants from NIHR Oxford BRC, grants from Medical Research Council (MR/S036741/1). Dr Carr reports grants from NIHR Biomedical Research Centre, grants from NIHR BioPatch i4i, grants from Wellcome Trust BioYarn and grants from Novartis. The views expressed are those of the author(s) and not necessarily those of the NHS, the NIHR, or the Department of Health.

Disclaimer The funding source played no role in the study design; in the collection, analysis, and interpretation of data; in the writing of the report; and in the decision to submit the article for publication. We confirm the independence of the researchers from funders and that all authors, external and internal, had full access to all of the data (including statistical reports and tables) in the study and can take responsibility for the integrity of the data and the accuracy of the data analysis. The views expressed are those of the author(s) and not necessarily those of the NHS, the NIHR, or the Department of Health.

Competing interests All authors have completed the ICMJE uniform disclosure form at www.icmje.org/coi_disclosure.pdf and declare: JAS reports personal fees from Biolacuna, personal fees from IP Asset Ventures, outside the submitted work; and he is part of the Carr Group at the University of Oxford, which is developing medical devices. He receives funding from the National Institute for Health Research (NIHR) Oxford Biomedical Research Centre (BRC). CH reports that he has received expenses and fees for his media work and expenses from the WHO. He has received financial remuneration from an asbestos case. He receives expenses for teaching EBM and is also paid for his GP work in NHS out of hours. He is Director of the CEBM at the University of Oxford, Editor in Chief of BMJ EvidenceBased Medicine and an NIHR Senior Investigator. AJC reports grants from Novartis, outside the submitted work. In addition, AJC has a patent for Oxford Yarn issued, and a patent for Oxford Patch issued.

Patient consent for publication Not required.

Provenance and peer review Externally peer reviewed.

Data availability statement Data are available in a public, open access repository. Data, code and the data extraction protocol are publicly available at https://osf.io/ g423d/.

Open access This is an open access article distributed in accordance with the Creative Commons Attribution 4.0 Unported (CC BY 4.0) license, which permits others to copy, redistribute, remix, transform and build upon this work for any purpose, provided the original work is properly cited, a link to the licence is given, and indication of whether changes were made. See: https://creativecommons.org/ licenses/by/4.0\%.

\section{ORCID iDs}

James Andrew Smith http://orcid.org/0000-0003-2634-0268

Roxanna E Abhari http://orcid.org/0000-0002-4520-6534

Zain Hussain http://orcid.org/0000-0002-0559-8289

Carl Heneghan http://orcid.org/0000-0002-1009-1992

Gary S Collins http://orcid.org/0000-0002-2772-2316

Andrew J Carr http://orcid.org/0000-0001-5940-1464

\section{REFERENCES}

1 Topol EJ. High-performance medicine: the convergence of human and artificial intelligence. Nat Med 2019;25:44-56.

2 Hwang TJ, Kesselheim AS, Vokinger KN. Lifecycle regulation of artificial Intelligence- and machine Learning-Based software devices in medicine. JAMA 2019. doi:10.1001/jama.2019.16842. [Epub ahead of print: 22 Nov 2019].

$3 \mathrm{He} \mathrm{J}$, Baxter SL, Xu J, et al. The practical implementation of artificial intelligence technologies in medicine. Nat Med 2019;25:30-6.

4 FDA. Proposed Regulatory Framework for Modifications to Artificial Intelligence/Machine Learning (Al/ML)-Based Software as a Medical Device (SaMD) - Discussion Paper and Request for Feedback; 2019.

5 Regulations.gov. Tips for submitting effective comments.

6 Office of the Commissioner, FDA. Comment on proposed regulations and submit Petitions, 2019. Available: http://www.fda.gov/regulatoryinformation/dockets-management/comment-proposed-regulationsand-submit-petitions

7 Regulations.gov - Docket Folder Summary. Available: https://www. regulations.gov/docket?D=FDA-2019-N-1185

8 R Core Team. R: a language and environment for statistical computing. R Foundation for Statistical Computing, 2019.

9 Kanter GP. Public comments and industry interests in Medicaid coverage decisions. JAMA Intern Med 2020;180:331-2.

10 Ahn R, Herrera-Perez D, Prasad V. Characteristics of public comments submitted to state health technology assessment programs in Oregon and Washington. JAMA Intern Med 2020;180:329-31.

11 Bowers S, Cohen D. How lobbying blocked European safety checks for dangerous medical implants. BMJ 2018;363:k4999.

12 Lin DH, Lucas E, Murimi IB, et al. Financial conflicts of interest and the centers for disease control and prevention's 2016 guideline for prescribing opioids for chronic pain. JAMA Intern Med 2017;177:427-8.

13 Pham-Kanter G. Revisiting financial conflicts of interest in FDA Advisory committees. Milbank Q 2014;92:446-70.

$14 \mathrm{Xu}$ J, Emenanjo O, Ortwerth M, et al. Association of appearance of conflicts of interest with voting behavior at FDA Advisory Committee Meetings-A cross-sectional study. JAMA Intern Med 2017;177:1038-40.

15 Hayes MJ, Prasad V. Financial conflicts of interest at FDA drug Advisory Committee meetings. Hastings Cent Rep 2018;48:10-13.

16 Bekelman JE, Li Y, Gross CP. Scope and impact of financial conflicts of interest in biomedical research: a systematic review. JAMA 2003;289:454-65.

17 Lundh A, Lexchin J, Mintzes B, et al. Industry sponsorship and research outcome. Cochrane Database Syst Rev 2017;2:MR000033.

18 Nagendran M, Chen Y, Lovejoy CA, et al. Artificial intelligence versus clinicians: systematic review of design, reporting Standards, and claims of deep learning studies. BMJ 2020;368:m689.

19 Zargar N, Carr A. The regulatory ancestral network of surgical meshes. PLoS One 2018;13:e0197883.

20 Institute of Medicine. Medical devices and the public's health: The FDA 510(k) clearance process at 35 years. Available: http://www. nationalacademies.org/hmd/Reports/2011/Medical-Devices-and-thePublics-Health-The-FDA-510k-Clearance-Process-at-35-Years.aspx

21 Lenzer J, ICIJ reporters. Medical device industry: international investigation exposes lax regulation. BMJ 2018;363:k4997. 